\title{
Trees and Nests: A Comparison between Two Hierarchical Metaphors in Educational Applications
}

\author{
Adi Katz \\ Department of Industrial Engineering and Management, Shamoon College of Engineering, Ashdod, Israel \\ Email: adis@sce.ac.il
}

Received December $2^{\text {nd }}, 2011$; revised January $6^{\text {th }}, 2012$; accepted January $17^{\text {th }}, 2012$

\begin{abstract}
The aim is to determine whether one of two hierarchical metaphors, the tree (parent-child) or the nested (object-container), is more suitable for designing educational interfaces for children. To cope with this issue an experimental educational application was designed with a prototype for each hierarchical metaphor. The application was evaluated in a laboratory experiment, where children participants interacted with the prototypes to find answers for questions that require searching for information. Task performance was measured in terms of effectiveness, efficiency and subjective aspects such as user perception of ease of use and user preference. The nested (object-container) metaphor was found to be preferred by users and superior in several objective parameters of performance efficiency, but no significant differences were found in the perceived ease of use and in the performance effectiveness. Implications for designing educational applications are discussed.
\end{abstract}

Keywords: Human Computer Interactions; Metaphoric Interfaces; Hierarchical Information Organization; Educational Applications; Usability; Children Interfaces Design

\section{Introduction}

Today, more than ever, children are exposed to computers in their early years of development and have easy access to computers and the Internet. Children use the Internet for schoolwork, playing games and communicating with each other, and are typically involved in activities that require searching and browsing for information (Hutchinson et al., 2006). Creating interfaces for young children presents particular challenges, and the designers of such applications must take into consideration that children are different than adults in the way they think and learn. An important requirement for an effective learning process is to foster a positive attitude and affect that nourish motivation, arouse curiosity, engage creativity, and turn the brain into an effective learning organism (Norman, 2004). It is most important that applications for children are provided with suitable interfaces that make both their learning process and their interaction with the application easy and enjoyable.

A successful user interface paradigm is to design the human-computer interaction based on metaphors already familiar from real life objects, actions and situations. A metaphoric system simply replicates the real life objects and portrays them in the computerized environment, so that the user can intuitively manipulate objects and work in a familiar manner. A suitable metaphor enables the user to function effectively in the new system, and helps overcome cognitive limitations in complex tasks (Te'eni et al., 2007). One of the implications of the cognitive load theory (CLT) in the area of instructional design is that the layout should be intuitive, so that the mind's capacity for processing information will not be overloaded and user activities will be focused on the concepts to be learned (Sorden, 2005). Instead of learning the system and its features, metaphorical designs free some of the users' limited cognitive recourses so they can be fully engaged and devoted to the data and the activities at hand. When cognitive resources are unnec- essarily devoted to mental activities such as retrieving from memory and learning new objects and actions, it may be at the expense of performance. Metaphors have a particularly important function in interfaces for young children (Ellis \& Blashki, 2001; Gilutz \& Black, 2010), are highly preferred by them and can induce curiosity and pleasure (De Angeli et al., 2006). A useful metaphor must be suitable for the user population (Katz \& Vaserman, 2009), and therefore, when designing for young users, the chosen metaphor must be borrowed from their everyday environment or conceptual world.

In order to cope with the overwhelming diversity of objects and properties in the world, people mentally group objects, treating them as instances of categories instead of as unique individuals. Hierarchical organization is one type of a natural evolution of a classification system in which people notice both distinctions and similarities among objects, and organize categories into hierarchies in which more specific classes are included in more general ones (Markman, 1989). In other words, many natural categories are hierarchically organized into nested class-inclusion relations, where some classes are super-ordinate or subordinate to others. For example: organisms/plants/flowers/Composite family/daisies/erbera daisies. Collins and Quillian's (1969) Hierarchical Network Model of semantic memory states that concepts are stored and represented as nodes within a hierarchical structure in our long-term memory (LTM), with meaningful associations between concepts. The "Is A" link is the most common link in this semantic network model.

Browsing for information is a methodical activity with a specific goal, which requires progressive filtering of results based on visual scanning and searching. Since hierarchical organization stems from greater accumulation of knowledge and experience (Markman, 1989), children have a difficulty dealing with hierarchies when searching and browsing. Children do not always navigate efficiently between categories, they may not 
think hierarchically, and may have a difficulty understanding abstract, top-level categories (Hutchinson et al., 2006). Metaphors may be a useful design paradigm to make it easier for children to cope with browsing through hierarchical levels of information. A metaphor based on a hierarchical organization that children are already familiar with, actually utilizes knowledge that has already been accumulated and this, as previously described, decreases the cognitive overload. Clearly, the chosen metaphor should also be appropriate for presenting the needed relationships between categories. For example, an application teaching organism hierarchies should present "Is-a" relationships (A grizzly bear Is-a bear and a bear Is-a land mammal).

A well-known system for organization and management of files and folders on our computing devices embodies two hierarchical metaphoric-based interfaces; one is the file manager interface, which reflects the tree metaphor, and the other is the desktop interface, which is based on a nested object-container metaphor. The tree metaphor layout that originates from the very first instantiations of computer desktop environments, has come out so pervasive that is firmly embedded into our intuitive ways of dealing with "items", be it images, videos or generally speaking any type of information that can be defined as “files" (Villegas, 2006). Users navigate by clicking on little structural elements (plus and minus symbols) that open and close various directories.

The nested (object-container) metaphor for hierarchical organization expresses the relationship of "object-within-object" and allows recursion to be visualized in an intuitively nested "Russian doll" fashion. The nested Russian doll is widely used in recursive problem solving and in programming and mathematics education to explain recursive models (Schiemenz, 2002). The most obvious example of the nested doll principle in computers is Microsoft Windows, where "windows" is simply windows inside windows inside windows.

The current research follows a previous study that compared the tree and the object-container metaphors in the forms of a family tree versus a cabinet of drawers-two alternative metaphoric designs to convey hierarchical learning of material. In the previous study no superiority of one metaphor over the other in the design of children's interfaces was found (Katz and Vaserman, 2009). Despite these results, we were still certain that a cabinet metaphor was more suitable for children since it inherits affordances that are the actions of organizing their belongings (toys, books, candy, etc.) in their natural environment at playrooms in their homes and kindergartens. Therefore the expectation was that this metaphor would be more intuitive and easy to operate, more usable and more preferable for young children than the tree metaphor. We believe that the previous results were obtained due to specific design elements of the metaphoric interface that caused an unwanted difference in usability between the tree and the cabinet designs. In addition it should be noted that the previous research was based on a small sample of only 10 subjects. In this research we decided to redesign the metaphors and test our hypotheses on a larger sample of subjects.

\section{Problem Definition and Solution}

Young children need encouragement, support and help in learning about the world and developing cognitively and emotionally. Pre-school children are at a very important stage of development in life, in which they are exposed to activities of the early learning of reading and writing. At this phase, most children are constantly involved in confident exploring behaviors, interacting with their environment in an active way and expanding their cognitive abilities on the basis of their own activities. We believe that educational tools such as learning applications for children should serve as a vehicle to promote learning, curiosity, exploratory behavior and independence in the learning process, and serve to flourish their knowledge. In addition, successive interactions and positive learning experiences at this stage in childhood can develop a high level of self confidence, esteem and efficacy. A sense of self efficacy has a positive influence on the success of the learning process, because previous successful and positive experiences develop expectations to succeed in similar tasks (Bandura, 1997). Self efficacy developing while interacting with an application used for learning gives children the confidence to continue learning with the same tool, and motivates them to explore additional tools and features.

Creating interfaces for young children presents particular challenges. Children have different cognitive capabilities than adults; they think and learn differently. The variable of age encompasses many critical developmental differences between childrens' and adults' ability to interact with technology, and their unique characteristics play an important role in creating a successful user experience for them. This is why usability testing techniques should be applied with a user centered design (UCD) approach, particularly when designing for children to meet their capabilities, needs and expectations (Gilutz \& Black, 2010).

The objectives for this research were to:

1) Design and implement the features of two hierarchical recursive metaphor prototypes for a computer program that educates children about animals: the tree and the nested metaphors.

2) Compare the two prototypes by conducting an experiment with pre-defined searching tasks, using subjective quantitative measures of the perceived ease of use (perceived usability) and user preference, as well as objective and quantitative measures of effective and efficient performance. In addition, gather qualitative data by directly observing the child-application interacttion.

\section{The Experiment}

The two hierarchical metaphors, tree and nested, were tested in a controlled experiment, in which children interacted with two metaphoric-based educational systems to answer questions regarding animals. The systems were aimed at the age group between four and six since most children at this age range are still unfamiliar with the file manager (tree) and nested-windows previously mentioned, and are therefore not influenced by conventional tree and nested metaphoric designs. This enabled testing the suitability of the metaphors without excessive noise. The metaphor design, tree versus closet, was manipulated to result in a natural tree design for the tree metaphor and a playroom closet design for the nested metaphor.

\section{The Dependent Variables}

The subjects' performance was observed in terms of efficiency and effectiveness, user usability perceptions, and preference. Table 1 presents the variables and how they were measured. 
Table 1.

The dependent variables and their measures.

\begin{tabular}{|c|c|}
\hline Dependent variables & Measure \\
\hline \multicolumn{2}{|l|}{ Performance } \\
\hline - Effectiveness & Overall number of correct answers \\
\hline - Efficiency & $\begin{array}{l}\text { - Overall task time } \\
\text { - Total number of clicks } \\
\text { - Total number of false clicks }\end{array}$ \\
\hline $\begin{array}{l}\text { Perceived ease of use } \\
\text { (usability) }\end{array}$ & $\begin{array}{l}\text { "How easy was it to search for answers in the } \\
\text { application?" (4-point Likert scale) }\end{array}$ \\
\hline Preference & $\begin{array}{l}\text { "Which application would you prefer having at } \\
\text { home: the tree or the closet?" }\end{array}$ \\
\hline
\end{tabular}

\section{The Independent and the Control Variables}

The independent variable was the metaphoric design of the interface: tree versus closet. The two designs are described in detail in Section “Apparatus-Metaphoric Interface Design”.

The controlled variables were the participant, the tasks and the order of task performance. The participants were thirty kindergarten children (twelve girls and eighteen boys), all within the target age group of four to six years old. The participants were classified as novice users in terms of Shneiderman's generic classification of users (Shneiderman, 1987) since they did not possess significant semantic knowledge of objects and actions in computers such as the hierarchical organization of files and folders, and lacked the syntactic knowledge of the metaphoric applications designed for this research. All participants interacted with both metaphoric educational applications at the kindergarten.

Each child had to carry out a series of search tasks to enable answering a set of questions, such as: "what is produced from tuna fish liver?”, and "how does a gorilla react to threat?” Overall there were fourteen questions regarding animals, with seven different tasks for each application. Two different but structurally and cognitively similar sets of tasks were created, with corresponding questions in each application. For example, a question appearing in the tree metaphor was: "what can be made of goose-feathers" and the corresponding question in the closet metaphor was: "what is produced from tuna fish liver".

In order to avoid possible bias due to the order of exposure of the subjects to each metaphor, the children were randomly divided into two groups of 15 children each, so that one group interacted with the tree prototype first and continued with the closet, and the second interacted in the opposite order (hereafter, $\mathrm{Tf}$ and Cf will be used as abbreviations for Tree first and Closet first, respectively). After having conducted seven search tasks in the first application, the applications were switched to perform the additional seven search tasks in the second application.

\section{Pilot Test}

After having implemented the two prototypes to meet the design criteria of the two hierarchical metaphors, both applications were pilot tested on six children as representatives of the potential users, to obtain feedback regarding the design, to identify potential usability problems, and to make sure that the experiment was not too difficult. The pilot participants were three girls and three boys between the ages 4.5 - 6. Each used both prototypes where three started with the tree metaphor and the other three started with the closet metaphor. The pilot was conducted so as to follow the user centered design (UCD) approach, to ensure the prototypes were compatible with the endusers' characteristics, such as cognitive development, cognitive limitations, preferences and other factors that may have affected the understandability and usability of the applications. The pilot participants' interaction with the applications was observed and the design was adjusted accordingly. UCD is extremely important when designing for children, and is widely used in the HCI literature in various variations such as cooperative inquiry and participatory and informant design (Baek \& Lee, 2003; Druin, 2005; Mazzone et al., 2008). The pilot resulted in some improvements in design features that were not easy or intuitive or caused confusion, and in several experimental changes.

\section{Apparatus-Metaphoric Interface Design}

The research was conducted in Israel, and therefore the interfaces' language was Hebrew. The two educational programs were identical in the type and amount of information, in the hierarchical categorization and levels (with "Is-a” relations between categories), and in icons and labels representing object categories.

Figure 1 presents the opening screens of the hierarchical metaphors: the tree metaphor on the left side, and the closet metaphor on the right. Figure 2 presents two compatible screens of the tree and the closet metaphors. The hierarchical classes that are displayed in both screens represent the following hierarchical levels: Mammals/Land Mammals/Ungulates/Horse; Deer; Cow; Donkey.

As aforementioned, specific design elements of the metaphoric interface have caused an unwanted difference in usability between the prototypes in the previous study comparing between the tree and cabinet metaphors (Katz \& Vaserman, 2009). The main problem was that the cabinet metaphor was inferior in usability characteristics not directly derived from the metaphor, such as a relative lack of information regarding the hierarchy in the visualization of hierarchical organization, and the use of a confusing navigational aid. Drawing conclusions from that research, great emphasis was placed on a unified transitioning between various hierarchical levels in both prototypes. This goal was accomplished by requiring an equal number of clicks to move from one level of the hierarchy to another, and designing each prototype to manifest the complete hierarchical path for each choice of the user.

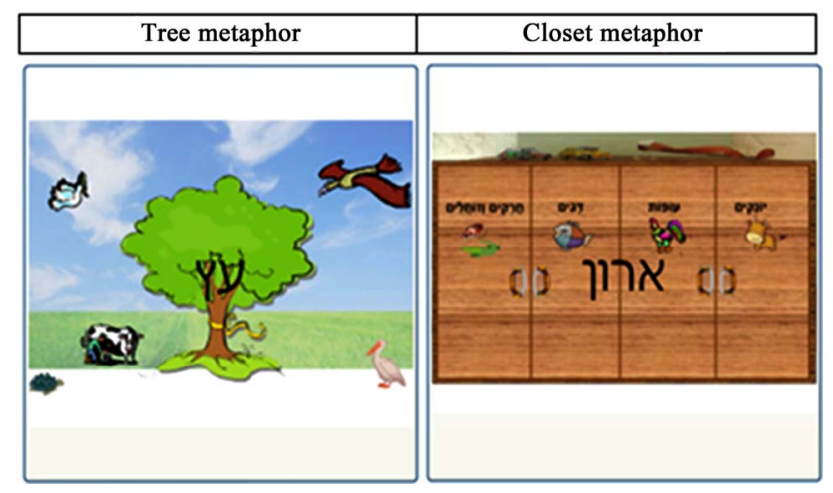

Figure 1.

The opening screens of each metaphoric interface. 
In the tree metaphor (Figure 2(a)), the four main (general) categories were displayed at the bottom part of the screen, closest to the tree's roots, and the highest branch displayed the most specific (leaf level) categories. This is obviously a natural way to present a physical tree that has a "bottom-up" development growing upwards from the roots in the ground. An alternative metaphoric design that utilizes the tree metaphor is the family tree (Katz \& Vaserman, 2009; Park \& Park, 2010), but it is typically drawn "top-down", presenting ancestors above their descendants. Note that both versions of the tree metaphor conceptually convey a "top-down" relation, in which categories start with the most general class, until reaching the lowest level of elementary subclass. The claim is that while adults distinguish between "top-down" and "bottom-up" in many areas that differentiate levels of abstraction, children are not aware of this "vertical flip" of real-life trees and hierarchical category development and therefore will find it easier to handle a hierarchical tree metaphor organizing information in a manner that imitates the natural growth of trees.

The tree metaphor endows the affordance of following paths from the root, and from one branch to another. Paths are revealed and concealed by unfolding and folding tree branches, respectively, by clicking on categorical nodes. Clicking on a node where the continuous branch seems hidden unfolds it above that node (in other words, expands the tree upwards), while clicking on an internal node folds the continuous branch back by hiding it. When clicking on another node, at the same or at an upper level in the hierarchy, the current node with an open continuous branch folds back to the level of the clicked node, and a new continuous branch unfolds at the clicked node. The reason for folding a branch when the user wishes to unfold another is to prevent a cognitive load that is likely to occur when too much and irrelevant information is exposed. We used navigation aids to highlight the hierarchical path, coloring the chosen nodes (categories) light blue, while leaving the other nodes (categories not on the path) in light green, and also by presenting the branches connecting chosen nodes in sharp colors, while the other branches in faded colors.

Following the idea of Katz and Vaserman (2009) of metaphoric designs for hierarchical organization for children, the cabinet metaphor used in the previous study was redesigned to a different variation of an object-container metaphor, which was named the closet metaphor (Figure 2(b)). The closet metaphor matches the typical organization of objects (such as books and toys) in children's playrooms. While the Katz and Vaserman (2009) cabinet metaphor strictly followed the recursive "Russian doll" fashion relations of doll within doll and so forth , by a cabinet of drawer within drawer and so forth, the newer closet metaphor contains different objects at different levels of the hierarchy, that can be placed one inside the other. The four main categories (see Figure 2(b)) are displayed as the closet's four doors. Opening each door reveals its nested drawers that contain boxes that contain animal cards representing the most specific (leaf level) categories. We believe that the current nested variation of the closet metaphor is more interesting and fun than the previous, and is a better imitation of a playroom closet that children are familiar with. Familiarity is an important design factor that has a strong impact on children: the more familiar is the interface metaphor, the better the comprehension achieved (Gilutz \& Black, 2010).

In Figure 2, one door-the mammals-is open to reveal two drawers, land mammals and sea mammals. The land mammals' drawer is also open to reveal four boxes nested inside it: carnivores, ungulates, rodents and haplorhini. The ungulates' box is open and exposes four cards for the following animals: horse, deer, cow and donkey. The closet-doors-drawers-boxes-cards relationships are in a nested "Russian doll" fashion: the biggest doll opens to reveal the next biggest doll, and so on until the tiniest doll is revealed, nested in all dolls together. Opening doors, drawers, and boxes (closed categories) is accomplished by intuitively clicking on their handles, and closing these objects is accomplished by clicking on them again. Clicking on an object that is closed (door, drawer or box) opens it and closes the object that was open so far. For example, in the current path presented in Figure 2, if one clicks on the sea mammal's drawer, it opens to reveal its boxes, and at the same time the land mammal's drawer closes. A click on the fish door reveals its drawers, and causes the mammal door to close. The closet metaphor endows a set of affordances that are extremely intuitive for young children: searching and revealing by opening and closing doors, drawers, and boxes.

In both designs, clicking on last level nodes (leaf level categories) opens an information window displaying an educational description of animal items, e.g. information about the deer or donkey. The children were required to access the information windows relevant to each task, to answer correctly. The number of levels and categories at each level of the hierarchy is adjusted according to a recommendation referring to $5 \pm 2$ categories in children's applications (Baek \& Lee, 2003).

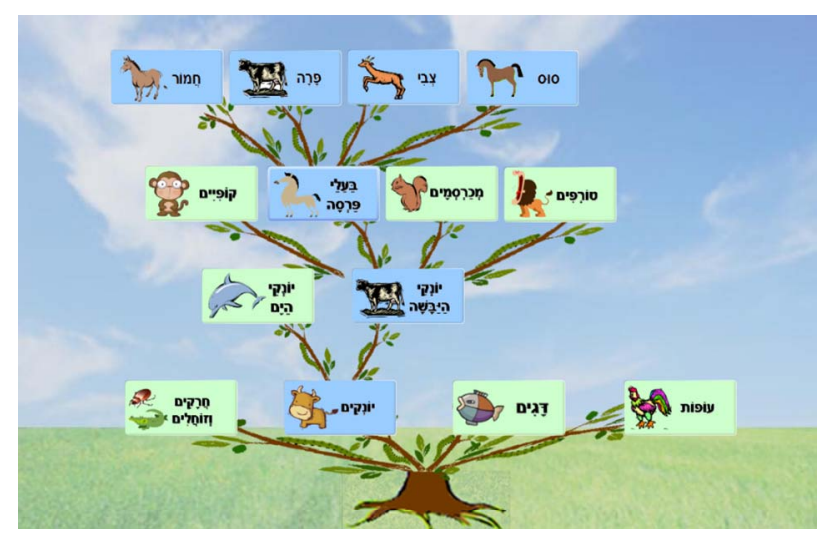

(a)

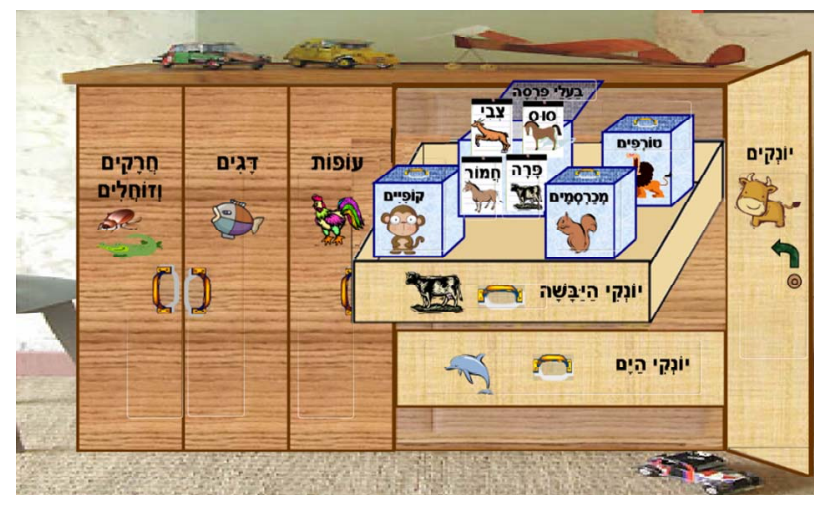

(b)

Figure 2.

screen shots of two compatible hierarchical paths for the two metaphors. (a) Tree metaphor; (b) Closet metaphor. 
Children need positive reinforcement and praise, which help them to experiment successfully (Yahaya \& Salam, 2009). To motivate the participants' involvement and arousal, we used positive reinforcement in the form of a glass candy jar that is gradually filled with a different candy for each task. Each time a child chooses the right question; a candy appears at the upper side of the screen and slowly falls down into the jar while playing a funny sound of applause and cries of glee ("correct!", "well done!”). Animation and sound effects are positive elements in designing for children (Gilutz \& Nielsen, 2007). Whenever a wrong answer is chosen, a frowning "smiley" appears in an animated entrance with an audio encouraging the child to try again. Audit output is crucial in interfaces designed for young children, to over come the lack of a reading ability. All text (displayed on buttons, information pages, instructions, etc.) is presented with punctuations as customary in Hebrew texts designated for young children, and is followed by a human voice that reads the text.

\section{Conducting the Experiment}

Each of the children participants interacted separately with both metaphors at a computer station in a quiet room at the kindergarten, under our supervision. As said, 15 children were randomly allocated to a group that interacted with the tree application first and the closet application second, and the other 15 children interacted with the closet application first and the tree application second. At the beginning of the interaction with each application, the children received a short tutorial about it, after which they practiced the use of the application by performing two preliminary search tasks prior to the actual experiment. The experiment itself was composed of fourteen tasks, seven in each application. After having conducted seven search tasks in the first application, the child performed the additional seven search tasks in the second application.

During the experimental phase, the applications' logs recorded the dependent variable measures of performance and at the end of the interaction with each application, a question reflecting the child's perceived ease of use of the application appeared on the screen ("How easy was it to search for answers in the application?"; the response was recorded on a 4 point Likert scale). At the end of the experiment, after interacting with both applications, a question regarding the child's application preference appeared ("Which application would you prefer to have at home: the tree or the closet?").

During the experimental phase qualitative data was gathered by direct observation of the child-application interaction, with a focus on gestures, facial cues and voice intonations. In addition the "think aloud" technique was used, by encouraging the children to speak out their thoughts, problems, indecisions and impressions. The information gathered from these observations added rich and useful information on the comprehensibility of the designs and user experience, as later discussed.

\section{Experimental Results}

We used $\mathrm{T}$ tests for paired (dependent) samples to test performance differences between the two prototypes: the tree versus the closet metaphor. The results are presented in Table 2. It can be seen that there is significant difference in efficiency in terms of overall task time, total number of false clicks, and total number of clicks, in favor of the Closet metaphor application; that is, it took considerably less time to answer the task questions, there were less false clicks and a smaller total number of clicks when using the Closet application. However, there is no significant difference in effectiveness in terms of the number of correct answers. Note that almost all children answered all the questions correctly with both applications.

A Wilcoxon signed ranks test was used to test the difference in user perceptions of the ease of use of the metaphors (perceived usability). The results indicate that there is no statistical difference in the perception of ease of use between the tree (Median $=2)$ and the closet (Median $=1)$ metaphors $(\mathrm{Z}=$ $-0.808, p=0.419)$. For both prototypes, most users stated that it was very easy or easy to use (26 and 28 for the tree and closet prototypes, respectively) Figure 3 presents user rankings of ease of use for each metaphor.

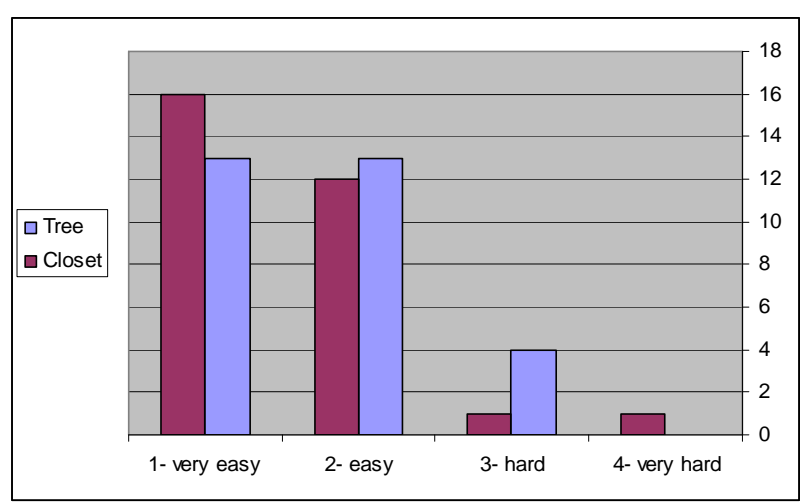

Figure 3.

Perceived ease of use of each metaphor.

Table 2.

Performance differences between the two interfaces.

\begin{tabular}{|c|c|c|c|c|c|c|c|}
\hline & \multirow{2}{*}{ Performance } & & \multicolumn{2}{|c|}{ Paired differences } & \multirow{2}{*}{$\mathrm{t}$} & \multirow{2}{*}{$\mathrm{df}$} & \multirow{2}{*}{ Sig. 2-tailed } \\
\hline & & & Mean & Std. D & & & \\
\hline \multirow{6}{*}{ Efficiency } & \multirow{2}{*}{$\begin{array}{l}\text { overall task } \\
\text { time }\end{array}$} & Tree & $11: 43$ & 3.11 & \multirow{2}{*}{-2.742} & \multirow{2}{*}{29} & \multirow{2}{*}{.010} \\
\hline & & Closet & $10: 41$ & 2.57 & & & \\
\hline & \# of false & Tree & 12.63 & 8.12 & \multirow{2}{*}{-3.249} & \multirow{2}{*}{29} & \multirow{2}{*}{.003} \\
\hline & clicks & Closet & 9.13 & 6.14 & & & \\
\hline & \multirow{2}{*}{$\begin{array}{l}\text { total \# of } \\
\text { clicks }\end{array}$} & Tree & 42.77 & 9.66 & \multirow{2}{*}{-2.452} & \multirow{2}{*}{29} & \multirow{2}{*}{.020} \\
\hline & & Closet & 39.46 & 7.77 & & & \\
\hline \multirow{2}{*}{ Effectiveness } & \multirow{2}{*}{$\begin{array}{l}\text { total \# of } \\
\text { correct answers }\end{array}$} & Tree & 6.60 & 0.62 & \multirow{2}{*}{1.153} & \multirow{2}{*}{29} & \multirow{2}{*}{0.258} \\
\hline & & Closet & 6.77 & 0.50 & & & \\
\hline
\end{tabular}


Of 30 children participants, 12 (40\%) preferred the tree prototype and $18(60 \%)$ preferred the closet. Six children said they preferred the closet because it was easier to use for finding information, 7 children said it was more fun to use, 3 said it was prettier and 5 subjects said they preferred the closet because they had a similar closet at home (some gave more than one explanation for their choice). Only one child said she preferred the tree because it was easier to use for finding information, 2 children said it was more fun to use, and 2 said it was prettier. Two subjects said they preferred the tree metaphor because they liked trees and nature. These preference explanations show that the metaphor evaluations were not only pragmatic but also hedonic in nature. This point is elaborated in the discussion.

The observations revealed that all children found interest in both applications, and their visible behavior and verbal expressions showed they were positively and happily engaged with them. There were no signs of discomfort, frustration or dissatisfaction, on the contrary: the children were highly motivated to find answers for the questions using both the tree and the closet metaphors. The conclusion is that both metaphors were comprehensible and satisfying.

\section{Discussion}

We were interested in determining whether one of two hierarchical metaphors, the tree or the closet was more suitable for the design of children's interfaces.

The results regarding interface efficiency, i.e., overall time to perform tasks, number of false clicks, and total number of clicks, show significant differences in favour of the closet metaphor interface. The overall task completion time and total number of clicks are related to Nielsen's "efficiency" dimension of usability, and the number of false clicks is related to the "few errors" dimension (1993). Therefore we can conclude that in efficiency performance parameters (expressing the resources consumed in the processes of achieving the task), the closet metaphor is more usable than the tree metaphor. However, in terms of performance effectiveness (i.e., the success of the task in terms of the number of correct answers to the questions), there was no difference between the two metaphoric designs.

Based on the results of $\mathrm{T}$ tests for independent samples to test the differences between the performances of the groups in the first and second exposure to the prototype, we can conclude that the order of exposure to the prototypes does not matter.

Referring to the test of user perception of the interfaces' ease of use, the results do not show a significant difference: both metaphorical interfaces were evaluated as easy or very easy to use. We expected the closet metaphor to be perceived as more usable for young children than the tree metaphor since it resembles the way they organize their belongings in their playrooms and therefore would be more intuitive. Overall, the results show that both metaphors are perceived usable for kindergarten children. Additional data, obtained from observations of the children while they interacted with the applications, showed that both metaphors were comprehensible, and there was no significant difference in the difficulty expressed in the action of transitioning between hierarchical levels.

The closet metaphor was designed to resemble any closet in the natural environment of children's playrooms. Choosing a metaphor that matches between the system and the real world of the user population is an important usability heuristic proposed by Nielsen (1994). The system should speak the user's language, with words and concepts that are familiar to the user, and should follow real-world conventions, presenting information in a natural and logical order. Most children have indeed preferred the closet metaphor. We believe that the current nested variation of the closet metaphor is more interesting and fun than the previous cabinet variation (Katz \& Vaserman, 2009), and is a better imitation of a playroom closet that children are familiar with. On the other hand, the tree metaphor seemed less familiar and was also more monotonous; using the same objects at each hierarchical level (nodes on paths that are made of branches). We refer to more heterogeneous design ideas for the tree metaphor later.

In summary, the results of the current study show superiority of the closet metaphor in user preference and in several objective parameters of performance efficiency, but not in the perceived usability or in performance effectiveness. Both metaphoric designs were effective in achieving the users' goals, and were similarly perceived as easy to use, but the closet metaphor stimulated a better user experience, leading the children to prefer it over the tree metaphor. User experience (UX) emphasizes the importance of understanding how the user feels about the system, in opposed to considering only the mere usability, with its emphasis on user performance. Although the main focus was to test the suitability of the metaphors in terms of usability, the results are in line with the shift from a cognitive and functional focus on computer applications towards an experiential affective view (Tractinsky et al., 2000; Dillon, 2001; De Angeli et al., 2002; Hassenzahl, 2003), and show that UX is extremely important . There are two distinct design objectives in HCI: one is optimizing user performance and the other is optimizing user satisfaction in achieving both pragmatic and hedonic goals (Bevan, 2009). We claim that UX is most essential in systems designed to foster a positive attitude in the learning process of young children. De Angeli et al. (2006) found that participants preferred an interface they evaluated as more engaging and fun, despite an acknowledged inferior usability. We agree that while it is indeed important to optimize user performance and to fulfill the pragmatic goals of the user, it is most important to optimize user hedonic goals as well.

Referring to the results that show superiority of the closet metaphor in efficiency but not in effectiveness, and also the result of superiority in user preference for the closet, we argue that while it is indeed important that the user accomplishes the end goal using a system, namely performance effectiveness (i.e., the quality of the task solution), the system should also address the process, namely performance efficiency (i.e., the resources consumed in the process of achieving the goal). The efficiency-effectiveness distinction is well known and respectively matches the process-outcome levels of user experience with technology (see for example Dillon, 2001). Yet, many studies measure only objective aspects of the process, overlooking the affective and emotional aspects and more attention should be given to user choice and preference. Dillon suggests the POA approach for viewing user experience, which introduces three levels of user interaction: Process (action), Outcome (result), and Affect (emotion), which deal respectively with what the user does, what he attains and how he feels (Dillon, 2001). It is reasonable to argue that users' evaluations of the system are influenced by the process of pursuing their goal, in terms of 
their enjoyment of the task and not only in terms of the time consumed or the level of task accomplished.

As described, this study is a follow up to a previous study that compared the tree and the nested metaphors (Katz \& Vaserman, 2009), but is not an exact replication of the comparison, especially because the sample size, age range and also the metaphoric designs have changed. Therefore we can not compare the results of the two researches and claim that one metaphor is more appropriate than the other for a given age range. A follow up study will be conducted to compare the current metaphoric designs of the natural tree versus the closet with children in the age range of the previous study (ages 7 - 11).

The study was limited to the comparison between two specific metaphoric designs. Although the closet metaphoric design was found to be superior in several important parameters, one should not conclude from the results that the tree metaphor is inappropriate. We stress that it is important not only to choose a good metaphor, but also to properly design its characteristics. The tree metaphor may be more successful if designed differently, and more creatively, perhaps with special interactive effects that are playful or humoristic (for example see De Angeli et al., 2006) or in the form of an online game that makes the learning experience more fun (see Park \& Park, 2010). Also, a tree metaphor that contains different objects at different hierarchy levels (such as branches, leafs, flowers and fruits) or alternatively having a gradual change of branch thickness (from the thickest to thinnest branches, imitating a real-world tree growth) may be more interesting and fun variations of the current. In addition, the tree metaphor may be more suitable than the closet for other tasks or for children at ages that we did not test.

\section{Conclusion}

We conclude that applying metaphors that are familiar to children from their real life environments is a successful user interface paradigm. We recommend that when designing an interface that can exploit alternative metaphoric ideas, one should test and compare them, by using the user centered design (UCD) approach to find out which design is better, which characteristics and features are more easy and intuitive to use, which are difficult, and how children react to each metaphor as whole. In addition, creating artifacts such as user interfaces is a very creative process, and since children are known as very creative, collaborating with them can inspire and empower adults with new insights to generate new ideas they would otherwise never have thought of. Working closely with children that represent the target audience as design partners in the application development process from the earliest stage of the design is a good strategy to create interfaces that are particularly suitable for them. Involving children in the design process, conducting user testing techniques that combine objective usability measures with subjective measures (such as user satisfaction, attitudes, feelings and preferences) and collecting qualitative data from observations are highly recommended as ways for optimizing both pragmatic and hedonic goals of the user.

\section{Acknowledgements}

We thank Ofir Marco and Ravid Kahalani for their contributions in designing the metaphors, planning of the experimental tasks, and running the pilot and the laboratory experiment.

\section{REFERENCES}

Bandura, A. (1997). Self-efficacy: The exercise of control. New York: W.H. Freeman.

Baek, J. S., \& Lee, K. P. (2003). A study of cognitive characteristics of children's information architecture using participatory design technique. Research Papers of Ergonomics for Children and Educational Environment, IEA Technical Committee.

Bevan, N. (2009). What is the difference between the purpose of usability and user experience evaluation methods? UXEM'09 Workshop, INTERACT 2009, Uppsala.

Collins A. M., \& Quillian M. R., (1969). Retrieval time from semantic memory. Journal of Verbal Learning and Verbal Behavior, 8, 240248. doi:10.1016/S0022-5371(69)80069-1

De Angeli, A., Lynch, P., \& Johnson, G. I. (2002) Pleasure versus efficiency in user interfaces: Towards an involvement framework. In W. S. Green, \& P. W. Jordan (Eds.), Pleasure with products: Beyond usability (pp. 97-111). London: Taylor \& Francis. doi:10.1201/9780203302279.ch7

De Angeli, A., Sutcliffe A., \& Hartmann, J. (2006). Interaction, usability and aesthetics: What influences users' preferences? Proceedings of the 6th Conference on Designing Interactive systems (DIS'06), New York, 271-280.

Dillon, A. (2001). Beyond usability: Process, outcome and affect in human-computer interactions. Canadian Journal of Library and Information Science, 26, 57-69. doi:10.1086/428691

Druin, A. (2005). What children can teach us: Developing digital libraries for children. Library Quarterly, 75, 20-41.

Ellis, K., \& Blashki. K. (2001). Producing an artifact as research: Multimedia for young children, 2001. Proceedings of the National Advisory Committee on Computing Qualifications, Napier, 259-264.

Gilutz, S., \& Black, J. B. (2010) Child and design factors interacting in children's HCI helping children focus on the content, not the interface. Designing for Children, 2-6 February 2010, Mumbai.

Gilutz, S., \& Nielsen, J. (2007). Usability of websites for children. USA: Nielsen Norman Group. URL (last checked 3 October 2011). http://www.designingforchildren.net/

Hassenzahl, M. (2003). The thing and I: Understanding the relationship between user and product. In M. Blythe, C. Overbeeke, A. Monk, \& P. C. Wright (Eds.), Funology: From usability to enjoyment (pp. 3142). Dordrecht: Kluwer.

Hutchinson, B. H., Bederson, B. B., \& Druin, A. (2006). The evolution of the international children's digital library searching and browsing interface. Proceedings of the 2006 Conference on Interaction Design and Children (IDC'06), (pp. 105-112). New York, NY: ACM.

Katz, A., \& Vaserman, D. (2009). A case study of two hierarchical recursive metaphors for information organization: The closet metaphor vs. the tree metaphor. Computer Modelling and New Technologies, 13, 7-17.

Markman, E. M. (1989). Categorization and naming in children: Problems of induction. Cambridge, MA: The MIT Press.

Mazzone, E., Read, C. J., Beale, R., Abuelmaatti, O., \& England, D. (2008). Understanding children's contributions during informant design. Proceedings of the 22nd British HCI Group Annual Conference (HCI 2008), Liverpool, 1-5 September 2008, 61-64.

Nielsen, J. (1993). Usability engineering. Boston: Academic Press.

Nielsen, J. (1994). Ten usability heuristics. URL (last checked 3 October 2011). http://www.useit.com/papers/heuristic/heuristic_list.html

Norman, D. (2004). Emotional design: Why we love (or hate) everyday things. New York: Basic Books.

Park, E. Y., \& Park, Y. H. (2010). A hierarchical interface design of a puzzle game for elementary education. International Journal of $u$ and e-Service, Science and Technology, 3, 43-50.

Schiemenz, B. (2002). Managing complexity by recursion. In R. Trappl (Ed.), Cybernetics and Systems (pp. 475-479). Wien: Austrian Society for Cybernetic Studies.

Shneiderman, B. (1987). Designing the user interface: Strategies for effective human-computer interaction. Boston: Addison-Wesley, Inc.

Sorden S. (2005). A cognitive approach to instructional design for multimedia learning. Informing Science Journal, 8, 263-279.

Te’eni, D., Carey, J., \& Zhang, P., (2007). Human computer interaction: 


\section{A. KATZ}

Developing effective organizational information systems. Hoboken: John Wiley \& Sons, Inc.

Tractinsky, N., Shoval-Katz, A., \& Ikar, D. (2000). What is beautiful is usable. Interacting with Computers, 13, 127-145.

doi:10.1016/S0953-5438(00)00031-X

Villegas, P., Concejero, P., Pérez, S., Prieto, J., Aragón, L., \& Diego, S. (2006). Human factors issues in 3D visualization module for multimedia collections in AceMedia. The 20th International Symposium on Human Factors in Telecommunication, Sophia-Antipolis, 20-23 March 2006.

Yahaya, W. A. J., \& Salam, S. N. A. (2009). Usability design strategies for children: Developing children learning and knowledge in decreasing children dental anxiety. Proceedings of the International Conference on Primary Education, Hong Kong, 25-27 November 2009. 